\title{
Compound climate risks in the COVID-19 pandemic
}

\author{
The COVID-19 pandemic will be an unprecedented test of governments' ability to manage compound risks, \\ as climate hazards disrupt outbreak response around the world. Immediate steps can be taken to minimize \\ climate-attributable loss of life, but climate adaptation also needs a long-term strategy for pandemic preparedness. \\ Carly A. Phillips, Astrid Caldas, Rachel Cleetus, Kristina A. Dahl, Juan Declet-Barreto, Rachel Licker, \\ L. Delta Merner, J. Pablo Ortiz-Partida, Alexandra L. Phelan, Erika Spanger-Siegfried, Shuchi Talati, \\ Christopher H. Trisos and Colin J. Carlson
}

T: he COVID-19 emergency has acutely overshadowed public reckoning with the climate crisis; the outbreak is still growing in most places, with over three million confirmed cases in 185 countries at the time of writing. The pandemic's disruption of daily lives, health systems and economies is unprecedented, and reverberations will continue long after the first wave of infections ebbs and a vaccine is developed. As outbreaks continue, governments will be faced with developing and adjusting policies that address not only the pandemic itself, but also potential collisions and intersections with other regional or global crises.

A concerning body of evidence already indicates that climate hazards, which are increasing in frequency and intensity under climate change, are likely to intersect with the COVID-19 outbreak and public health response. These compound risks will exacerbate and be exacerbated by the unfolding economic crisis and long-standing socioeconomic and racial disparities, both within countries and across regions, in ways that will put specific populations at heightened risk and compromise recovery. These burdens will fall disproportionately on countries in the Global South; United Nations Secretary-General Guterres has noted that "....as with the climate crisis, the African continent could end up suffering the greatest impacts [of the COVID-19 pandemic]" 1 .

This poses a tremendous policy challenge: while keeping climate goals and pandemic containment in sight, countries will face a drumbeat of climate adaptation crises that require immediate response and, ideally, advance preparation.

\section{The compound risks}

Only a few months after the start of the COVID-19 pandemic, several climate hazards have collided with the outbreak

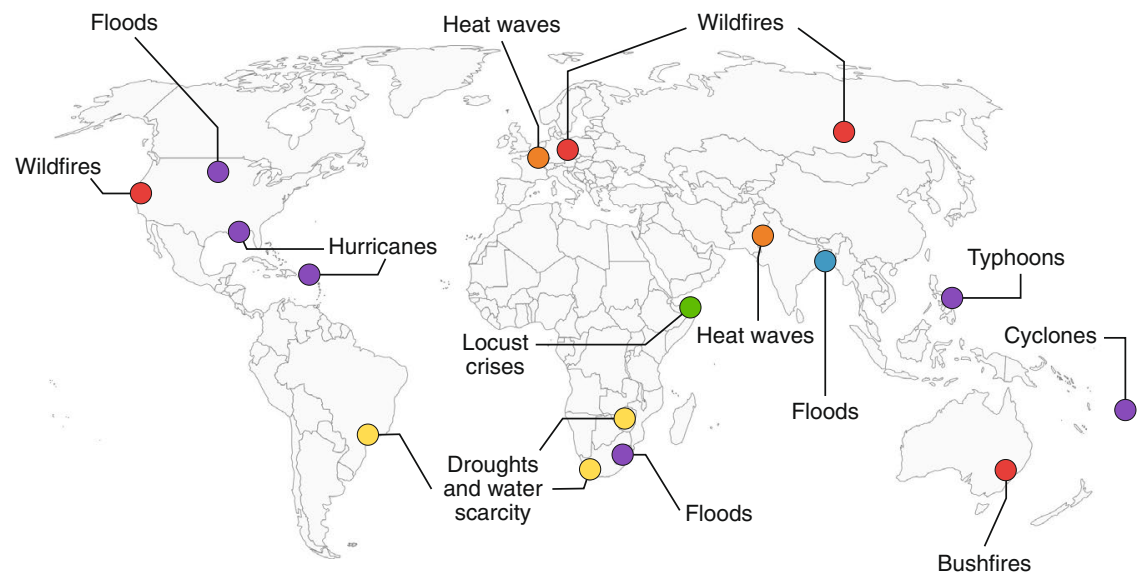

Fig. 1 | Likely upcoming climate hazards during the COVID-19 pandemic. Climate-attributable risks are likely to intersect with the COVID-19 crisis all around the world, with many already causing disruptions or likely to do so over the next 12 to 18 months.

itself or jeopardized sensitive public health work. While the specific nature of threats will change over seasons and regions (Fig. 1), the overall problem is universal and should be seen as a sobering signal of what lies ahead for countries all over the world.

Storms, flooding and droughts are among the most immediately apparent sources of displacement and disruption under the pandemic. Fiji was hit by a category 5 tropical cyclone during the onset of the outbreak; Vanuatu, the Solomon Islands and Tonga will also face challenges to recovery under states of emergency while preventing introduction of the virus ${ }^{2}$. The Gulf Coast of the USA is bracing for a severe hurricane season while the state of Louisiana has become a national hotspot of COVID-19 mortality, in part due to a legacy of racial discrimination and underinvestment in healthcare. In South Africa, local authorities are struggling with how to maintain social distancing during flooding in informal settlements where the policy is already extremely difficult to implement. In Zimbabwe, drought has left millions without access to clean water and at risk of acute food insecurity during June-September 2020 (ref. $^{3}$ ). These impacts, as well as the power cuts associated with drought in southern African countries heavily dependent on hydropower ${ }^{4}$, will affect these countries' response to the virus.

Furthermore, this upcoming year may be the hottest on record ${ }^{5}$. Extreme heat events, like those already experienced this year in Florida and the southwestern USA, may lead to particularly high excess mortality and morbidity, and will likely disrupt power supplies, hospitals and emergency services, especially in cities. This is likely to severely impact persons already at high risk of heat-related illness, including older adults, those living in poverty (especially those who cannot afford air conditioning), those with pre-existing health conditions, incarcerated people, the shelterless and persons with mobility challenges or disabilities. Wildfires 
will also intersect with the COVID-19 response in the western USA over the coming months, especially as the pandemic disrupts wildfire prevention measures and firefighter training programs $s^{6}$. This challenge is already apparent in Australia, where lasting health impacts from air pollution caused by an extreme bushfire season will likely exacerbate COVID-19 morbidity ${ }^{7}$, and the outbreak will further strain healthcare and the economy.

On an even broader scale, a combination of climate and geopolitical stressors are driving forced migrations ${ }^{8}$. Displaced populations, like those residing in refugee camps, are also uniquely at risk from COVID-19 outbreaks because of living conditions that are incompatible with social distancing and a lack of access to testing or healthcare. Pandemic-related border closures further exacerbate these challenges.

\section{Policy actions to limit loss of life today}

In this rapidly unfolding crisis, governments, health agencies and disease experts must take immediate action to confront COVID-19, but must also tackle inevitable climate-related disasters to help minimize loss of life. Emergency response agencies and first responders are particularly likely to find themselves deployed across multiple crises simultaneously, putting them under unprecedented strain. As an example, in the USA, the Federal Emergency Management Agency (FEMA) is now tasked with coordinating the nationwide COVID-19 response as well as any response to ongoing extreme weather and climate-related disasters, all while understaffed and under-resourced to do so.

Coordination is needed at every level of government to prevent potential conflicts of strategy across agencies, sectors and scales. For example, this spring, communities in the Midwestern USA have faced the prospect of evacuation due to flooding at the same time as stay-at-home orders have been in place ${ }^{9}$, and local emergency responders are urging social distancing. In these and other exposed communities (for example, hurricane zones or wildfire regions), difficult policy decisions lie ahead, including whether and how hospitals - especially intensive care units - can be evacuated safely, and how to manage broader health risks in the event of large-scale emergency sheltering needs ${ }^{10}$. Given that federal disaster response will likely prioritize evacuation and will leave local efforts to contain the resulting surges in COVID-19 cases, issuing formal updates to COVID-19 response guidance for state and local authorities (for example, covidlocal.org) will be critical.
In addition to acute emergency responses, policy measures are needed that ensure continuity of basic services. Ensuring continuity of electricity, water and other utilities will be critical to limit loss of life during heat waves, wildfires and hurricanes ${ }^{11}$. Similarly, when water agencies shut off service for non-payment of bills - a situation more likely to affect those who live in poverty or have lost jobs during the current economic crisis hand-washing becomes infeasible. In areas of the Global South, wealthy countries must provide budget support both to maintain these basic services in the near term and to invest in climate-resilient infrastructure to manage long-term risk.

Countries will need to develop detailed plans for compound risk preparedness, taking account of regional differences in climate vulnerability, the strength of existing health and social safety net systems, and outbreak trajectory. In all cases, governments and multilateral institutions responding to COVID-19, the climate crisis and their intersection must recognize that interventions and guidance must be tailored to the unique vulnerabilities, needs and circumstances of affected populations.

\section{A pandemic preparedness strategy for climate adaptation}

The climate adaptation community must develop a long-term strategy for pandemic preparedness $^{12,13}$, as COVID-19 is neither the first nor only time that our globalized society will face these types of compound risks; for example, Puerto Rico was forced to stop Zika surveillance and response in the aftermath of Hurricane Maria. Current governance and institutional structures - and the risk frameworks used in the IPCC and National Climate Assessment reports - are vulnerable to compartmentalization, especially in the health sector. More interdisciplinary, cross-sectoral risk assessments are needed, including planning for low-probability, high-impact events. These assessments must explicitly consider spatial and temporal coincidence of physical hazards and health or socioeconomic risk factors, interdependencies between sectors (for example, the food-energy-water-health nexus) and the potential for feedback loops. Solutions must similarly be more integrated and robust, taking into account interactions, trade-offs and co-benefits across sectors and at different scales - and therefore across traditional jurisdictions of government agencies - under a range of scenarios.

On the climate resilience front, many countries, including the USA, continue to address worsening impacts through the highly inadequate frame of disaster response. This means that there is still far too little emphasis on pre-disaster preparedness, and that the same agencies and resources that manage disasters are forced into attempting to build resilience to climate change on an ad hoc basis. Prepositioning adequate technical assistance and funding, and committing to automatic escalators in aid to cope with acute crises, is vital. One successful example is the Famine Early Warning System Network (FEWS NET), a collaborative effort among USAID, several US science agencies (NOAA, NASA, USGS and USDA), and 28 countries to track conditions - like the COVID-19 crisis - that could lead to acute food crises. Programs like FEWS NET exemplify multinational cooperation that is informed by scientific projections, uses a systems approach to develop solutions and helps ensure ambitious action on a scale commensurate with global risks. However, as the COVID-19 pandemic has demonstrated, technical core capacities alone are insufficient for preparedness and resilience: any climate adaptation framework must prioritize equitable outcomes and entrench good governance and accountability.

Furthermore, structural racism and systemic inequities put people of colour and the economically vulnerable at heightened risk in the face of both climate change and pandemics, a situation that we are already seeing unfold. One policy to address the structural inequalities highlighted under climate change and the COVID-19 outbreak is affordable universal health coverage that rectifies inequitable access to both preventative (addressing potential comorbidities during pandemics and climate-related disasters) and acute (ensuring rapid treatment during emergencies) care. In many places, the current overlapping crises are laying bare pre-existing gaps in healthcare and social services that must be addressed. Achieving the World Health Organization's goal of global universal health coverage by 2030 is critical to reduce health system vulnerability and to minimize long-term health impacts of climate-attributable events. In some countries, policy steps taken during COVID-19 may lay the groundwork for permanent expansion of healthcare in this direction; already, Ireland has nationalized healthcare for the duration of the pandemic. Solutions like these that centre equity, justice and human rights will ensure resilience in both climate change adaptation and pandemic preparedness. 
Beyond universal health coverage and proactive climate resilience measures, both climate change adaptation and pandemic preparedness can be framed as part of countries' legal obligation to realize the right to health through their laws, policies and budgets. Under international human rights instruments, such as the International Covenant on Economic, Social and Cultural Rights (1966), countries have obligations to respect, protect and fulfil the right to health, including taking steps to prevent epidemic disease ${ }^{14}$. There is increasing recognition that addressing climate change is a component of realizing the right to health: the Paris Agreement acknowledges that in taking action to address climate change, countries should consider, respect and promote their obligations on the right to health. Robust global cooperation and governance with a human rights-centred approach - supported by appropriate legal and institutional frameworks - is a prerequisite for successfully confronting these multi-dimensional, overlapping challenges with integrated solutions.
Carly A. Phillips (D) 1 , Astrid Caldas (D),

Rachel Cleetus ${ }^{1}$, Kristina A. Dahl',

Juan Declet-Barreto', Rachel Licker',

L. Delta Merner'1, J. Pablo Ortiz-Partida (iD

Alexandra L. Phelan², Erika Spanger-Siegfried',

Shuchi Talati', Christopher H. Trisos (D) ${ }^{3,4}$ and

\section{Colin J. Carlson (D) 2 的}

${ }^{1}$ Union of Concerned Scientists, Cambridge, MA, USA. ${ }^{2}$ Center for Global Health Science and Security, Georgetown University Medical Center, Washington D.C., WA, USA. ${ }^{3}$ African Climate and Development Initiative, University of Cape Town, Cape Town, South Africa. ${ }^{4}$ Centre for Statistics in Ecology and the Environment, University of Cape Town, Cape Town, South Africa.

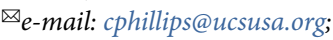

colin.carlson@georgetown.edu

Published online: 15 May 2020

https://doi.org/10.1038/s41558-020-0804-2

\section{References}

1. Secretary-General's remarks at virtual meeting with the African Group. United Nations https://www.un.org/sg/en/content/ sg/statement/2020-04-15/secretary-generals-remarks-virtualmeeting-the-african-group (2020).

2. Tropical Cyclone Harold challenges disaster and public health management. World Meteorological Organization https://public. wmo.int/en/media/news/tropical-cyclone-harold-challengesdisaster-and-public-health-management (2020).

3. Zimbabwe famine early warning systems network. FEWS NET https://fews.net/southern-africa/zimbabwe (2020).

4. Conway, D. et al. Nat. Clim. Change 5, 837-846 (2015).

5. State of the Climate: Global Climate Report for March 2020 (NOAA, 2020); https://www.ncdc.noaa.gov/sotc/global/202003/ supplemental/page-2

6. Groom, N. Trump administration halts wildfire prevention tool in California over coronavirus. Reuters https://www. reuters.com/article/us-health-coronavirus-usa-wildfires/ trump-administration-halts-wildfire-prevention-tool-incalifornia-over-coronavirus-idUSKCN21X1HD (2020).

7. Wu, X., Nethery, R. C., Sabath, M. B., Braun, D. \& Dominici, F. Preprint at https://www.medrxiv.org/content/10.1101/2020.04.05. 20054502v2 (2020).

8. Abel, G. J., Brottrager, M., Cuaresma, J. C. \& Muttarak, R. Glob. Environ. Change 54, 239-249 (2019).

9. Dahl, K. A. Continued social distancing critical for U. S. regions where flooding and COVID-19 are set to collide. Union of Concerned Scientists https://blog.ucsusa.org/kristy-dahl/ continued-social-distancing-critical-for-us-regions-whereflooding-and-covid-19-are-set-to-collide (2020).

10. Graeden, E., Carlson, C. J. \& Katz, R. Lancet Glob. Health https://doi.org/10.1016/S2214-109X(20)30191-1 (2020).

11. Graff, M. \& Carley, S. Nat. Energy https://doi.org/10.1038/s41560020-0625-6 (2020).

12. Marco, M. D. et al. Proc. Natl Acad. Sci. USA 117, 3888-3892 (2020).

13. Carlson, C. J. et al. Preprint at https://www.biorxiv.org/content/10 1101/2020.01.24.918755v1 (2020).

14. International Covenant on Economic, Social, and Cultural Rights (United Nations, 1966); https://www.ohchr.org/en/ professionalinterest/pages/cescr.aspx

\title{
Climate-related financial disclosures in the public sector
}

\author{
Expectations for the Task Force on Climate-related Financial Disclosure's framework to drive climate action in \\ the private sector are high, and there is growing interest in its relevance for guiding public sector climate action. \\ However, consideration of the framework's limitations is critical prior to public sector application.
}

\section{lan Edwards, Kiri Yapp, Sam Mackay and Brendan Mackey}

$\Lambda$ Task Force on Climate-related Financial Disclosures (TCFD) was established by the G20's Financial Stability Board on the premise that more complete and consistent disclosure of climate-related risks would encourage better decision-making and risk management by entities, resulting in a more stable financial system $^{1}$. The expectation is that disclosure by entities of their climate-related financial risks will enable the collective market to incorporate such risks into pricing decisions, in turn imposing the market discipline needed to transition towards achieving low carbon and climate-resilient targets.

The TCFD released its final recommendations in June 2017, to widespread attention and support. Premised on the disclosure of four core elements - governance, strategy, risk management, and metrics and targets - the TCFD recommendations extend existing frameworks by recommending disclosure of material climate-related risks, risk governance and management processes, and resilience of the business strategy under different climate-related scenarios. By February 2020, the TCFD had drawn over 1,037 proclaimed supporters, including NGOs, corporations and stock exchanges ${ }^{2}$.

Government support of the TCFD recommendations has predominantly taken the form of financial regulator endorsement and consideration of associated regulation for their use in private sector disclosures, and considering regulation for the same. However, this support has extended to consideration of the framework's application to the public sector itself. For example, the city of Vancouver included TCFD-aligned disclosures in its 2018 annual financial report as one mechanism for mainstreaming climate risk considerations into the city's processes. Further, legislation introduced by the New Zealand government in 2019 compels organizations, including the public service and local authorities, to provide disclosures on climate change adaptation consistent with the core elements of the TCFD.

While the uptake of the TCFD among both regulators and businesses represents positive progress on climate action, application of the framework without consideration of its limitations risks suboptimal results for the private and public sectors alike. 